\title{
Periodic Solutions to Non-Autonomous Second-Order Dynamical Systems
}

\author{
An-Min Mao, Miao-Miao Yang \\ School of Mathematical Sciences, Qufu Normal University, Qufu, China \\ E-mail:maoam@163.com \\ Received January 17, 2011; revised March 7, 2011; accepted March 12, 2011
}

\begin{abstract}
We study the multiple existence of periodic solutions for a second-order non-autonomous dynamical systems $\ddot{u}+\nabla_{u} V(t, u)=0(1)$. Using the method of invariant sets of descending flow and chain of rings theorem, we obtain the existence of seven $2 \pi$-periodic solutions.
\end{abstract}

Keywords: Critical Point, Invariant Sets of Descending Flow, (PS) Condition

\section{Introduction}

In this paper, we consider the existence of seven nontrivial solutions for the second order non-autonomous systems

$$
\ddot{u}+\nabla_{u} V(t, u)=0 . \quad t \in[0,2 \pi], \quad u \in R^{N} .
$$

For each $u \in R^{N}$ the function $V(t, u)$ is periodic in $t$ with period $2 \pi$.

Problem (1) has been studied by many authors and there is a large literature, see, for example, [1-11] (cf. the references quoted in them).

Using the method of invariant sets of descending flow, Z. Liu and J. Sun [1] got at least four periodic solutions of (1). Via the variational method, which has been mostly used to prove the existence of solutions of (1), M. Willem, J. Mawhin, S. Li, M. Schechter, C. Tang and others proved existence under various conditions (cf. the reference given in these publications). Also, the fixed point theorems in cones can be chosen to establish the existence of solutions for (1), see [12].

The goal of this paper is to find more periodic solutions for problem (1). We get at least seven periodic solutions of (1) by using the method of invariant sets of descending flow and Chain of rings Theorem, which is obtained in [7].

Let us give some notations. For two functions $u$ and $v$ defined on $[0,2 \pi]$ and taking their values in $R^{N}$, we define a partial order by $u \leq v$ if and only if $u_{i}(t) \leq v_{i}(t)$ for all $t \in[0,2 \pi]$ and $i=1,2, \cdots, N$. If $u_{i}(t)<v_{i}(t)$ for all $t \in[0,2 \pi]$ and $i=1,2, \cdots, N$, the relationship between $u$ and $v$ will be denoted by $u \ll v$.

(H1) There are two couples of functions $\phi, \varphi, \alpha, \beta$ $\in C^{2}\left(R, R^{N}\right)$ with period $2 \pi$ such that:

$$
\phi \ll \psi, \alpha \ll \beta, \phi \not \subset \beta, \alpha \not \leq \psi,
$$

and

$$
\begin{aligned}
-\ddot{\phi} & \leq \nabla_{u} V(t, \phi), \\
-\ddot{\psi} & \geq \nabla_{u} V(t, \psi), \\
-\ddot{\alpha} & \leq \nabla_{u} V(t, \alpha), \\
-\ddot{\beta} & \geq \nabla_{u} V(t, \beta),
\end{aligned}
$$

for any $i=1,2, \cdots, N$, there is $t_{i} \in[0,2 \pi]$ such that

$$
-\ddot{\phi}_{i}\left(t_{i}\right)<\frac{\partial}{\partial u_{i}} V\left(t_{i}, \phi\left(t_{i}\right)\right),
$$

for any $i=1,2, \cdots, N$, there is $\tilde{t}_{i} \in[0,2 \pi]$ such that

$$
-\ddot{\psi}_{i}\left(\tilde{t}_{i}\right)>\frac{\partial}{\partial u_{i}} V\left(\tilde{t}_{i}, \psi\left(\tilde{t}_{i}\right)\right),
$$

for any $i=1,2, \cdots, N$, there is $t_{i}^{\prime} \in[0,2 \pi]$ such that

$$
-\ddot{\alpha}_{i}\left(t_{i}^{\prime}\right)<\frac{\partial}{\partial u_{i}} V\left(t_{i}^{\prime}, \alpha\left(t_{i}^{\prime}\right)\right),
$$

for any $i=1,2, \cdots, N$, there is $t_{i}^{\prime \prime} \in[0,2 \pi]$ such that

$$
-\ddot{\beta}_{i}\left(t_{i}^{\prime \prime}\right)>\frac{\partial}{\partial u_{i}} V\left(t_{i}^{\prime \prime}, \beta\left(t_{i}^{\prime \prime}\right)\right) .
$$

(H2) There exists a constant $K>0$ such that every entry of the matrix $\nabla_{u u} \boldsymbol{V}(t, u)+K^{2} \boldsymbol{I}$ is nonnegative if $u$ is a function satisfying $u \geq \phi$ or $u \leq \psi$ or $u \geq \alpha$ or $u \leq \beta$, where $\nabla_{u u} \boldsymbol{V}(t, u)$ is the Hessian matrix of 
$\boldsymbol{V}$ and $\boldsymbol{I}$ is the $\boldsymbol{N} \times \boldsymbol{N}$ unit matrix.

(H3) There exists $\mu>2$ and $R>0$ such that, for $|u| \geq R$,

$$
0<\mu V(t, u) \leq u \nabla_{u} V(t, u),
$$

where $|u|$ means the Euclidean norm of $u$ in $R^{N}$.

Theorem 1. If (H1), (H2) and (H3) are satisfied, then problem (1) has at least seven periodic solutions.

Remark 1.1. Conditions in Theorem 1 can be satisfied by generic functions. As examples, it can be proved that

$$
V=\sum_{i=1}^{N} u_{i}^{4}+2 \sum_{i \neq j} u_{i} u_{j}+\frac{2 N}{1+|u|^{2}}
$$

satisfies all the conditions in Theorem 1. One should take $\phi \equiv u \ll 0$ and $\psi \equiv v \gg 0$, in which $u_{1}=u_{2}=\cdots=u_{N}$, $v_{1}=v_{2}=\cdots=v_{N},|u|$ and $|v|$ are sufficiently small. $\alpha$ and $\beta$ can be chosen in the same way.

Remark 1.2. Our work is based on the results in $[1,7]$.

\section{Preliminary and Lemmas}

Let $H$ be the Hilbert space of vector functions $u(t)$ having period $2 \pi$ and belonging to $H^{1}$ on $[0,2 \pi]$, with the following inner product

$$
(u, v)=\int_{0}^{2 \pi}\left(\dot{u} \dot{v}+K^{2} u v\right) \mathrm{d} t,
$$

where $K$ is a fixed number satisfying (H2). The corresponding norm in $H$ is denoted by $\|\cdot\|_{H}$ and

$$
\|u\|_{H}^{2}=(u, u)=\int_{0}^{2 \pi}\left(|\dot{u}|^{2}+K^{2} u^{2}\right) \mathrm{d} t .
$$

Let $X$ be the Banach space of $N$-vector functions $u(t)$ having period $2 \pi$ and belonging to $C^{1}$ on $[0,2 \pi]$ and $X$ is continuously imbedded in $H$.

Define a functional $J: H \rightarrow R$ as

$$
J(u)=\int_{0}^{2 \pi}\left[\frac{1}{2}|\dot{u}|^{2}-V(t, u)\right] \mathrm{d} t .
$$

Then the critical points of $J$ correspond to the solutions of problem (1). Here $\tilde{K}:=\left\{u \in H \mid J^{\prime}(u)=0\right\} \subset X$. We have

$$
J^{\prime}(u)=u-\left(-\frac{\mathrm{d}^{2}}{\mathrm{~d} t^{2}}+K^{2}\right)^{-1}\left(\nabla_{u} V(t, u)+K^{2} u\right)
$$

here $\left(-\frac{\mathrm{d}^{2}}{\mathrm{~d} t^{2}}+K^{2}\right)^{-1}$ is the inverse operator of $-\frac{\mathrm{d}^{2}}{\mathrm{~d} t^{2}}+K^{2}$ with the periodic condition of period $2 \pi$.

Denote

$$
A u=\left(-\frac{\mathrm{d}^{2}}{\mathrm{~d} t^{2}}+K^{2}\right)^{-1}\left(\nabla_{u} V(t, u)+K^{2} u\right) .
$$

Now we will explain that (2) holds: Noting that

$$
\left(J^{\prime}(u), v\right)=\int_{0}^{2 \pi}\left[\dot{u} \dot{v}-\nabla_{u} V(t, u) v\right] \mathrm{d} t,
$$

and

$$
(A u, v)=\int_{0}^{2 \pi}\left(A \dot{u} \dot{v}+K^{2} A u v\right) \mathrm{d} t,
$$

then

$$
\begin{aligned}
(u, v)-(A u, v) & =\int_{0}^{2 \pi}\left(\dot{u} \dot{v}+K^{2} u v-A \dot{u} \dot{v}-K^{2} A u v\right) \mathrm{d} t \\
& =\int_{0}^{2 \pi} \dot{u} \dot{v}-\left[\left(-\frac{d^{2}}{d t^{2}}+K^{2}\right) A u-K^{2} u\right] v \mathrm{~d} t
\end{aligned}
$$

For $A u=\left(-\frac{\mathrm{d}^{2}}{\mathrm{~d} t^{2}}+K^{2}\right)^{-1}\left(\nabla_{u} V(t, u)+K^{2} u\right)$, we have

$$
\left(J^{\prime}(u), v\right)=(u, v)-(A u, v) .
$$

For $u_{0} \in X$, consider the initial value problem

$$
\frac{\mathrm{d} u(\tau)}{\mathrm{d} \tau}=-u(\tau)+A u(\tau), u(0)=u_{0}
$$

both in $H$ and in $X$. Let $u\left(\tau, u_{0}\right)$ and $\tilde{u}\left(\tau, u_{0}\right)$ be the unique solution of (3) in $H$ and in $X$ respectively, with maximal right existence interval $\left[0, \eta\left(u_{0}\right)\right)$ and $\left[0, \tilde{\eta}\left(u_{0}\right)\right)$.

Lemma 1. (Lemma 5.1 in [1]) $\eta\left(u_{0}\right)=\tilde{\eta}\left(u_{0}\right)$ and $u\left(\tau, u_{0}\right)=\tilde{u}\left(\tau, u_{0}\right)$ for all $0 \leq \tau<\eta\left(u_{0}\right)$. Moreover, if $\lim _{\tau \rightarrow \eta\left(u_{0}\right)} u\left(\tau, u_{0}\right)=u^{*}$ in the $H$ topology for some $u^{*} \in \tilde{K}$, the critical set of $J$, then the limit is also valid in the $X$ topology.

Definition 1. (Chain of rings) (Definition 5 in [7]) Assume that $D_{1}, D_{2}, \cdots, D_{n}(n \geq 2)$ all are nonempty path connected sets. When $n \geq 4, D_{1}$ intersects only with $D_{2}$ and $D_{n}, D_{n} \cap D_{1}=A_{n} . D_{i}$ intersects only with $D_{i-1}, D_{i+1}(i=2,3, \cdots, n-1)$, and $D_{i} \cap D_{i+1}=A_{i}$ $(i=1,2, \cdots, n-1)$, we say that $D_{1}, D_{2}, \cdots, D_{n}$ form a chain of rings. For the case of $n=3, D_{1} \cap D_{2}=A_{1} \neq \varnothing$, $D_{2} \cap D_{3}=A_{2} \neq \varnothing, \quad D_{3} \cap D_{1}=A_{3} \neq \varnothing$, and $D_{1} \cap D_{2}$ $\cap D_{3}=\varnothing$, we say that $D_{1}, D_{2}, D_{3}$ form a chain of rings. For the case of $n=2, \quad D_{1} \cap D_{2}=A_{1} \cup A_{2}, A_{1} \cap A_{2}$ $=\varnothing$, we say that $D_{1}$ and $D_{2}$ form a chain of rings.

Lemma 2. (Theorem 4 and Remark 5 in [7]) Assume that $H$ is a Hilbert space, $J \in C^{1}\left(H, R^{1}\right), \quad J^{\prime}(u)$ $=u-A u, \forall u \in H, J$ satisfies the (PS) condition on $H$. $D_{1}, D_{2}, \cdots, D_{n} \quad(n \geq 3)$ are open convex subsets of $X$, and form a chain of rings. $A\left(\partial_{X} D_{i}\right) \subset D_{i}$ $(i=1,2, \cdots, n)$,

1) when $n$ is even, if

$$
\inf _{\substack{\frac{n}{2} \\ u \in \mathcal{U}_{j=1}^{X} \bar{A}_{2 j}^{X}}} J(u)>-\infty \quad \text { or } \inf _{\substack{\frac{n}{2} \\ u \in \mathcal{U}_{j=1} \bar{A}_{2 j-1}^{X}}} J(u)>-\infty,
$$


then $J$ has at least $\frac{3}{2} n+1$ critical points;

2) when $n$ is odd, if

$$
\inf _{u \in \cup \frac{n+1}{2} \bar{A}_{2 j-1}^{X}} J(u)>-\infty \quad \text { or } \inf _{\substack{\frac{n-1}{2} \\ \sum_{j=1}^{2}} \bar{A}_{2 j}^{X} \cup \bar{A}_{1}^{X}} J(u)>-\infty,
$$

then $J$ has at least $\frac{3}{2}(n-1)+3$ critical points.

\section{Proof of Theorem 1}

We now give the proof of Theorem 1 .

Proof. Step 1. First we will prove that $J$ satisfies (PS) condition.

(H3) implies the existence of constants $C_{1}>0$ and $\mathrm{C}_{2}>0$ such that

$$
V(t, u) \geq C_{1}|u|^{\mu}-C_{2}, \quad \forall u \in R^{N} .
$$

Actually let $g(x)=V(t, x u)$,

$$
\begin{aligned}
\frac{\mathrm{d} g(x)}{\mathrm{d} x} & =\nabla_{u} V(t, x u) u=\frac{1}{x} \nabla_{u} V(t, x u) x u \\
& \geq \frac{\mu}{x} V(t, x u)=\frac{\mu}{x} g(x),
\end{aligned}
$$

i.e. $\frac{\mathrm{d} g(x)}{g(x)} \geq \frac{\mathrm{d} x}{x} \mu$. For $x \geq 1$, we get

$$
\begin{gathered}
\int_{1}^{x} \frac{\mathrm{d} g(s)}{g(s)} \geq \int_{1}^{x} \frac{\mu}{s} \mathrm{~d} s, \\
\ln |g(x)|-\ln |g(1)| \geq \ln |x|^{\mu}-0, \\
|g(x)| \geq|g(1)| \cdot|x|^{\mu}, \\
|V(t, x u)| \geq|V(t, u)| \cdot|x|^{\mu} .
\end{gathered}
$$

thus,

$$
\begin{aligned}
|V(t, u)| & =\left|V\left(t,|u| \frac{u}{|u|}\right)\right| \geq\left|V\left(t, \frac{u}{|u|}\right)\right| \cdot|u|^{\mu} \\
& \geq\left|\min _{t \in[0,2 \pi]} V(t, \pm 1)\right| \cdot|u|^{\mu}:=C_{1}|u|^{\mu} .
\end{aligned}
$$

For $x<1$, one has

$$
\int_{1}^{x} \frac{\mathrm{d} g(s)}{g(s)} \geq \int_{x}^{1} \frac{\mu}{s} \mathrm{~d} s .
$$

Similarly,

$$
\begin{gathered}
|g(x)| \leq|g(1)| \cdot|x|^{\mu}, \\
|V(t, x u)| \leq|V(t, u)| \cdot|x|^{\mu} .
\end{gathered}
$$

then

$$
\begin{aligned}
|V(t, u)| & =\left|V\left(t,|u| \frac{u}{|u|}\right)\right| \leq\left|V\left(t, \frac{u}{|u|}\right)\right| \cdot|u|^{\mu} \\
& \leq\left|\max _{t \in[0,2 \pi]} V(t, \pm 1)\right| \cdot|u|^{\mu}:=C_{2} .
\end{aligned}
$$

If $|u|<R$, by the continuousness of $V$, we can take proper $C_{2}$ such that (4) holds.

For $|u| \geq R$, by (H3) and (4), it follows that, for $u \in H$,

$$
\begin{aligned}
& J(u)-\frac{1}{2}\left(J^{\prime}(u), u\right) \\
& =\int_{0}^{2 \pi}\left[\frac{1}{2}|\dot{u}|^{2}-V(t, u)\right] \mathrm{d} t-\frac{1}{2} \int_{0}^{2 \pi}\left[|\dot{u}|^{2}-\nabla_{u} V(t, u) u\right] \mathrm{d} t \\
& =\int_{0}^{2 \pi}\left[-V(t, u)+\frac{u}{2} \nabla_{u} V(t, u)\right] \mathrm{d} t \\
& \geq\left(\frac{\mu}{2}-1\right) \int_{0}^{2 \pi} V(t, u) \mathrm{d} t \geq\left(\frac{\mu}{2}-1\right) \cdot C_{1} \int_{0}^{2 \pi}|u|^{\mu} \mathrm{d} t-C_{3} .
\end{aligned}
$$

For $|u|<R$, by the continuousness of $-V(t, u)$ $+\frac{u}{2} \nabla_{u} V(t, u)$, we can take proper $C_{3}$ such that

$$
J(u)-\frac{1}{2}\left(J^{\prime}(u), u\right) \geq\left(\frac{\mu}{2}-1\right) \cdot C_{1} \int_{0}^{2 \pi}|u|^{\mu} \mathrm{d} t-C_{3}, \forall u \in H .
$$

Similarly, we can obtain that

$$
\begin{aligned}
& J(u)-\frac{1}{\mu}\left(J^{\prime}(u), u\right) \\
& =\int_{0}^{2 \pi}\left[\frac{1}{2}|\dot{u}|^{2}-V(t, u)\right] \mathrm{d} t-\frac{1}{\mu} \int_{0}^{2 \pi}\left[|\dot{u}|^{2}-\nabla_{u} V(t, u) u\right] \mathrm{d} t \\
& =\left(\frac{1}{2}-\frac{1}{\mu}\right) \int_{0}^{2 \pi}|\dot{u}|^{2} \mathrm{~d} t+\int_{0}^{2 \pi}\left[-V(t, u)+\frac{u}{\mu} \nabla_{u} V(t, u)\right] \mathrm{d} t \\
& =\left(\frac{1}{2}-\frac{1}{\mu}\right) \int_{0}^{2 \pi}|\dot{u}|^{2}+K^{2}|u|^{2} \mathrm{~d} t-\left(\frac{1}{2}-\frac{1}{\mu}\right) \int_{0}^{2 \pi} K^{2}|u|^{2} \mathrm{~d} t \\
& \quad+\int_{0}^{2 \pi}\left[-V(t, u)+\frac{u}{\mu} \nabla_{u} V(t, u)\right] \mathrm{d} t \\
& \geq\left(\frac{1}{2}-\frac{1}{\mu}\right)\|u\|_{H}^{2}-\left(\frac{1}{2}-\frac{1}{\mu}\right) K^{2} \int_{0}^{2 \pi}|u|^{2} \mathrm{~d} t .
\end{aligned}
$$

From these inequalities, we see that, if $\left\{u_{n}\right\}_{1}^{\infty} \subset H$ such that $\left|J\left(u_{n}\right)\right|<C$ and $J^{\prime}\left(u_{n}\right) \rightarrow 0$ as $n \rightarrow \infty$, then by (5), one has

$$
\begin{gathered}
\left(\frac{\mu}{2}-1\right) \cdot C_{1} \int_{0}^{2 \pi}\left|u_{n}\right|^{\mu} \mathrm{d} t-C_{3} \leq C+1+\left\|u_{n}\right\|_{H}, \\
\int_{0}^{2 \pi}\left|u_{n}\right|^{\mu} \mathrm{d} t \leq C_{4}+C_{5}\left\|u_{n}\right\|_{H}
\end{gathered}
$$


By (6) and (7), we obtain

$$
\begin{gathered}
\left(\frac{1}{2}-\frac{1}{\mu}\right)\left\|u_{n}\right\|_{H}^{2}-\left(\frac{1}{2}-\frac{1}{\mu}\right) K^{2} \int_{0}^{2 \pi}\left|u_{n}\right|^{2} \mathrm{~d} t \leq C+1+\left\|u_{n}\right\|_{H}, \\
\left\|u_{n}\right\|_{H}^{2} \leq C_{6}+C_{7} \int_{0}^{2 \pi}\left|u_{n}\right|^{2} \mathrm{~d} t+\left\|u_{n}\right\|_{H}
\end{gathered}
$$

and

$$
\begin{aligned}
& \int_{0}^{2 \pi}\left|u_{n}\right|^{2} \mathrm{~d} t \leq\left(\int_{0}^{2 \pi} 1^{\frac{\mu}{\mu-2}} \mathrm{~d} t\right)^{\frac{\mu-2}{\mu}}\left(\int_{0}^{2 \pi}\left(\left|u_{n}\right|^{2}\right)^{\frac{\mu}{2}} \mathrm{~d} t\right)^{\frac{2}{\mu}} \\
& =C_{8}\left(\int_{0}^{2 \pi}\left|u_{n}\right|^{\mu} \mathrm{d} t\right)^{\frac{2}{\mu}} \leq C_{8}\left(C_{4}+C_{5}\left\|u_{n}\right\|_{H}\right)^{\frac{2}{\mu}} \\
& \leq C_{8}\left(C_{4}+1+C_{5}\left\|u_{n}\right\|_{H}\right)^{\frac{2}{\mu}} \leq C_{8}\left(C_{4}+1+C_{5}\left\|u_{n}\right\|_{H}\right)
\end{aligned}
$$

It follows from (8) and (9) that

$$
\left\|u_{n}\right\|_{H}^{2} \leq C_{9}+C_{10}\left\|u_{n}\right\|_{H},
$$

and here $C_{4} \sim C_{10}$ are positive constants.

(10) implies that $\left\{u_{n}\right\}$ is bounded in $H$ and therefore it has a subsequence, relabeled $\left\{u_{n}\right\}$, weakly convergent in $H$ and strongly convergent in $L^{p}$ $\left(1 \leq p<2^{*}\right)$. Thus

$$
\left(J^{\prime}\left(u_{n}\right)-J^{\prime}(u), u_{n}-u\right) \rightarrow 0 \text { as } n \rightarrow \infty,
$$

and

$$
\begin{aligned}
& \left|\int_{0}^{2 \pi}\left(\nabla_{u} V\left(t, u_{n}\right)-\nabla_{u} V(t, u)\right)\left(u_{n}-u\right) \mathrm{d} t\right| \\
& \leq\left\|\nabla_{u} V\left(t, u_{n}\right)-\nabla_{u} V(t, u)\right\|_{L^{q}}\left\|u_{n}-u\right\|_{L^{p}} \rightarrow 0 \text { as } n \rightarrow \infty \\
& \quad\left(J^{\prime}\left(u_{n}\right)-J^{\prime}(u), u_{n}-u\right) \\
& =\left\|\dot{u}_{n}-\dot{u}\right\|_{L^{2}}^{2}+\int_{0}^{2 \pi}\left(\nabla_{u} V\left(t, u_{n}\right)-\nabla_{u} V(t, u)\right)\left(u_{n}-u\right) \mathrm{d} t
\end{aligned}
$$

then we have

$$
\left\|\dot{u}_{n}-\dot{u}\right\|_{L^{2}}^{2} \rightarrow 0 \text { as } n \rightarrow \infty .
$$

by

$$
\left\|u_{n}-u\right\|^{2}=\left\|\dot{u}_{n}-\dot{u}\right\|_{L^{2}}^{2}+\left\|u_{n}-u\right\|_{L^{2}}^{2}
$$

and

$$
\left\|u_{n}-u\right\|_{L^{2}} \rightarrow 0 \text { as } n \rightarrow \infty
$$

we get

$$
\left\|u_{n}-u\right\| \rightarrow 0 \text { as } n \rightarrow \infty .
$$

Step 2. Let

$$
\begin{aligned}
& D_{1}=\{u \in X \mid u \ll \psi\}, D_{2}=\{u \in X \mid u \gg \phi\}, \\
& D_{3}=\{u \in X \mid u \gg \alpha\}, D_{4}=\{u \in X \mid u \ll \beta\},
\end{aligned}
$$

It is easy to see that $D_{1} \sim D_{4}$ are all open convex subsets of $X$, and

$$
\begin{aligned}
& D_{1} \cap D_{2}=A_{1} \neq \varnothing, D_{2} \cap D_{3}=A_{2} \neq \varnothing, \\
& D_{3} \cap D_{4}=A_{3} \neq \varnothing, D_{4} \cap D_{1}=A_{4} \neq \varnothing .
\end{aligned}
$$

Since $\phi \not \leq \beta, \quad \alpha \not \notin \psi$, we have $D_{1} \cap D_{3}=\varnothing$, $D_{2} \cap D_{4}=\varnothing$.

Hence $\left\{D_{i}\right\}_{1}^{4}$ form a chain of rings.

If $u \in \partial_{X} D_{1}$, then $u \leq \psi$. Condition (H2) implies that

$$
\begin{aligned}
& \left(\nabla_{u} V(t, \psi)+K^{2} \psi\right)-\left(\nabla_{u} V(t, u)+K^{2} u\right) \\
& =\int_{0}^{1}\left(\nabla_{u u} V(t, u+s(\psi-u))+K^{2} I\right)(\psi-u) d s \geq 0 .
\end{aligned}
$$

Note that $\left(-\frac{d^{2}}{d t^{2}}+K^{2}\right)^{-1} u=\int_{0}^{2 \pi} k(\cdot, s) u(s) \mathrm{d} s$, where

$$
k(t, s)=\left\{\begin{array}{l}
\frac{\cosh ((t-s-\pi) K)}{2 K \sinh (\pi K)}, 0 \leq s \leq t \leq 2 \pi ; \\
\frac{\cosh ((s-t-\pi) K)}{2 K \sinh (\pi K)}, 0 \leq t \leq s \leq 2 \pi .
\end{array}\right.
$$

Since $k(t, s)>0$, for any $0 \leq t, s \leq 2 \pi$, we have

$$
\begin{aligned}
A \psi-A u= & \left(-\frac{d^{2}}{d t^{2}}+K^{2}\right)^{-1} \\
& \cdot\left(\nabla_{u} V(t, \psi)+K^{2} \psi-\nabla_{u} V(t, u)-K^{2} u\right) \geq 0 .
\end{aligned}
$$

And by (H1)

$$
\begin{aligned}
\psi-A \psi= & \left(-\frac{d^{2}}{d t^{2}}+K^{2}\right)^{-1} \\
& \cdot\left(\left(-\frac{d^{2}}{d t^{2}}+K^{2}\right) \psi-\nabla_{u} V(t, \psi)-K^{2} \psi\right) \geq 0 .
\end{aligned}
$$

The maximum principle shows that $\psi \gg A \psi$. Therefore $A u \ll \psi$ for all $u \in \partial_{X} D_{1}$, that is $A u \in D_{1}$ for all $u \in \partial_{X} D_{1}$. Hence $A\left(\partial_{X} D_{1}\right) \subset D_{1}$. In a similar way, $A\left(\partial_{X} D_{i}\right) \subset D_{i}$, for $i=2,3,4$.

Since

$$
\begin{aligned}
& \bar{A}_{1}^{X}=\bar{D}_{1}^{X} \cap \bar{D}_{2}^{X} \subset\{u \in X \mid \phi \leq u \leq \psi\}, \\
& \bar{A}_{3}^{X}=\bar{D}_{3}^{X} \cap \bar{D}_{4}^{X} \subset\{u \in X \mid \alpha \leq u \leq \beta\},
\end{aligned}
$$

and $J$ is bounded on a bounded set, we get

$$
\inf _{\bar{D}_{1}^{X} \cap \bar{D}_{2}^{X}} J(u)>-\infty \text {, and } \inf _{\bar{D}_{3}^{X} \cap \bar{D}_{4}^{X}} J(u)>-\infty .
$$

From Lemma 5 and Remark 1, we know that $J$ has at least $\frac{3}{2} \times 4+1=7$ critical points.

Remark 3.1. If (H1) (H2) and the following condition 
are satisfied.

(H4) There exists $R_{1}>0$ and positively definite constant matrixes $\boldsymbol{A}$ and $\boldsymbol{B}$ with $\boldsymbol{A} \boldsymbol{B}=\boldsymbol{B} \boldsymbol{A}$ such that

$$
\boldsymbol{A} \leq \nabla_{u u} V(t, u) \leq \boldsymbol{B}, \forall u \in R^{N},|u| \geq R_{1} .
$$

Since $\boldsymbol{A B}=\boldsymbol{B} \boldsymbol{A}$, there is an orthogonal matrix $\boldsymbol{T}$ such that $\boldsymbol{T A} \boldsymbol{T}^{\prime}=\boldsymbol{T} \boldsymbol{B} \boldsymbol{T}^{\prime}$ are simultaneously diagonal matrixes. Let $\boldsymbol{T A} \boldsymbol{T}^{\prime}=\operatorname{diag}\left\{\alpha_{1}, \cdots, \alpha_{N}\right\}$ and $\boldsymbol{T} \boldsymbol{B} \boldsymbol{T}^{\prime}=$ $\operatorname{diag}\left\{\beta_{1}, \cdots, \beta_{N}\right\}$ and assume also that $\alpha_{i}>0$ for $i=1,2, \cdots, N$ and that

$$
\left(\cup_{i=1}^{N}\left[\alpha_{i}, \beta_{i}\right]\right) \cap\left\{n^{2} \mid n=0,1,2, \cdots\right\}=\varnothing .
$$

Then (1) has at least seven periodic solutions.

[1] shows $J(u)$ satisfies the (PS) condition under (H4). From the proof of Theorem 1, we can get this conclusion.

\section{Acknowledgements}

We should like to express our appreciation to the referees for suggesting how to improve our paper.

\section{References}

[1] Z. Liu and J. Sun, "Invariant Sets of Descending Flow in Critical Point Theory with Applications to Nonlinear Differential Equations," Journal of Differential Equations, Vol. 172, No. 2, 2001, pp. 257-299. doi:10.1006/jdeq.2000.3867

[2] M. Schechter, "Periodic Non-Autonomous Second-Order Dynamical Systems," Journal of Differential Equations, Vol. 223, No. 2, 2006, pp. 290-302. doi:10.1016/j.jde.2005.02.022

[3] Z. Y. Wang, J. H. Zhang and Z. T Zhang, "Periodic Solutions of Second Order Non-Autonomous Hamiltonian Systems with Local Superquadratic Potential," Nonlinear Analysis: Theory, Methods \& Applications, Vol. 70, No.
10, 2009, pp. 3672-3681. doi:10.1006/jmaa.1995.1002

[4] S. J. Li and M. Willem, "Applications of Local Linking to Critical Point Theory," Journal of Mathematical Analysis and Applications, Vol. 189, No. 1, 1995, pp. 6-32.

[5] A. Ambrosetti and P. H. Rabinowitz, "Dual Variational Methods in Critical Point Theory and Applications," Journal of Functional Analysis, Vol. 14, No. 4, 1973, pp. 349-381. doi:10.1016/0022-1236(73)90051-7

[6] J. X. Sun and J. L. Sun, "Existence Theorems of Multiple Critical Points and Applications," Nonlinear Analysis, Vol. 61, No. 8, 2005, pp. 1303-1317. doi:10.1016/j.na.2005.01.082

[7] J. R. Graef, L. J. Kong and H. Y. Wang, "Existence, Multiplicity and Dependence on a Parameter for a Periodic Boundary Value Problem," Journal of Differential Equations, Vol. 245, No. 5, 2008, pp. 1185-1197. doi:10.1016/j.jde.2008.06.012

[8] F. Zhao and X. Wu, "Existence and Multiplicity of Periodic Solutions for Non-Autonomous Second-Order Systems with Linear Nonlinearity," Nonlinear Analysis, Vol. 60, No. 2, 2005, pp. 325-335.

[9] J. Mawhin and M. Willem, "Critical Point Theory and Hamiltonian Systems," Springer, Berlin, New York, 1989.

[10] P. H. Rabinowitz, "Minimax Methods in Critical Point Theory with Applications to Differential Equations," Expository Lectures from the CBMS Regional Conference, Series in Mathematics, American Mathematical Society, Vol. 65, 1986.

[11] C. L. Tang, "Periodic Solutions of Nonautonomous Second-Order Systems with Sublinear Nonlinearity," Proceedings of the American Mathematical Society, Vol. 126, No. 11, 1998, pp. 3263-3270. doi:10.1090/S0002-9939-98-04706-6

[12] H. Y. Wang, "Periodic Solutions to Non-Autonomous Second-Order Systems," Nonlinear Analysis: Theory, Methods \& Applications, Vol. 71, No. 3-4, 2009, pp. 1271-1275. 